\title{
Cryogenic Thermal Absorptance Measurements on Small-Diameter Stainless Steel Tubing
}

James Tuttle ${ }^{\mathrm{a}}$, Amir Jahromi, Edgar Canavan, and Michael DiPirro

NASA Goddard Space Flight Center, Code 552, Greenbelt, MD 20771 USA

${ }^{a}$ Corresponding author: james.g.tuttle@nasa.gov

\begin{abstract}
The Mid Infrared Instrument (MIRI) on the James Webb Space Telescope includes a mechanical cryocooler which cools its detectors to their 6 Kelvin operating temperature. The coolant gas flows through several meters of small-diameter stainless steel tubing, which is exposed to thermal radiation from its environment. Over much of its length this tubing is gold-plated to minimize the absorption of this radiant heat. In order to confirm that the cryocooler will meet MIRI's requirements, the thermal absorptance of this tubing was measured as a function of its environment temperature. We describe the measurement technique and present the results.
\end{abstract}

Keywords: Absorptance, cryocooler, radiation 


\section{INTRODUCTION}

The Mid Infrared Instrument (MIRI) on the James Webb Space Telescope (JWST) includes detectors which will operate at approximately six Kelvin. These detectors will be cooled by Joule-Thomson (JT) expansion of helium gas, which will be pre-cooled to 18 Kelvin by a pulse-tube cryocooler. The 18 Kelvin helium will flow to the JT valve over a length of several meters through a small, stainless steel tube. This tube is surrounded by a thermal environment with complex view-factors and expected temperatures as high as 120 Kelvin. To ensure the required performance of the cooling system, it is critical to minimize the heat absorbed by the helium over this length. Significant engineering effort has been spent in developing and characterizing a lowthermal-conductance structural support system for the tube. In addition, the tube has been gold-plated over much of its length to keep its thermal absorptance low and reduce radiative heating from its surroundings.

A finite element thermal model including the entire JWST spacecraft predicts that the tube will absorb a sufficiently small amount of heat, and the cooling system will meet its performance requirements. However, this result depends on the thermal absorptance of the gold-plated tube falling below a certain expected maximum value at its 18 Kelvin operating temperature. To reduce the uncertainty in this model prediction, it was desirable to directly measure the thermal radiation power absorbed by this tubing per unit length as a function of the radiative source temperature. We describe below a test that was carried out by suspending a length of the tube inside a blackbody cavity and measure the power it absorbed as a function of the cavity temperature.

\section{THEORY}

The radiative heat exchange between an isothermal blackbody cavity and a relatively small object suspended inside the cavity involves some subtle details. The object's surfaces are exposed to radiation with a blackbody power spectrum characteristic of the cavity's temperature, $T_{B}$. The object absorbs some of this radiation, and the rest is reflected back and absorbed entirely by the cavity walls. The fraction of this radiation that is absorbed by the small object is the total hemispheric absorptance, $\alpha$, which is a function of $T_{B}$ as well the object's own temperature, $T_{S}$, surface texture, and material properties. The object also emits an amount of thermal radiation dependent on its surface area, its total hemispheric emissivity $\varepsilon$, and $T s$. In general $\varepsilon$ may not be equal to $\alpha$, but both depend on the same characteristics of the object. However, $\varepsilon$ is strictly a property of the object and is totally independent of $T_{B}$. Expressed as an equation, the net heat (in Watts) absorbed by the object is

where $A$ is the object's surface area (in $\left.\mathrm{m}^{2}\right)$, and $\sigma=5.67 \times 10^{-8}\left(\mathrm{~W} / \mathrm{m}^{2} / \mathrm{K}^{4}\right)$ is the StefanBoltzmann constant. When the sample and source temperatures are nearly equal, their radiation spectra are approximately proportional to each other. In this case $\alpha=\varepsilon$, and the radiative heat exchange is referred to as "grey-body."

The reflection of radiation at a smooth interface between dielectric materials is described by the Fresnel Equations. For the special case of monochromatic polarized 
infrared radiation incoming through a vacuum and reflecting off a smooth metallic surface, certain approximations are valid, and the reflectance equations are:

$$
\begin{aligned}
& R_{p}=\frac{\frac{60}{60}+2 \sqrt{\frac{30}{\frac{30}{2}}} \cos +\cos ^{2}}{\cos \cos ^{2}} \\
& R_{n}=\frac{\frac{60}{60} \cos ^{2} 2 \sqrt{\frac{30}{6}} \cos +1}{\frac{6 \cos ^{2}+2 \sqrt{\frac{30}{2}} \cos +1}{}} .
\end{aligned}
$$

Here the polarizations are parallel and normal to the surface, and $\lambda, \beta$, and $\rho$ are the wavelength $(\mathrm{cm})$, incidence angle (relative to normal), and electrical resistivity (ohm$\mathrm{cm})$, respectively ${ }^{1}$. For un-polarized radiation, the reflectivity $\operatorname{RTot}(\lambda, \beta, \rho)$ is the average of these polarized values, and the absorptance $\alpha_{T o t}(\lambda, \beta, \rho)=1-R_{\text {Tot. }}$ For a given wavelength and resistivity, the hemispheric absorptance is given by

$$
{ }_{H}=2{\underset{0}{2}}_{T o t}^{\overline{2}}(, \quad,) \cos () \sin () d .
$$

Using a program such as Mathematica ${ }^{\mathrm{TM}}$, this integral can be performed analytically, resulting in

$$
\begin{gathered}
{ }_{H}=\frac{2}{n}+4 n \frac{3}{4}+\tan ^{1} 1+\frac{1}{n} \div+\tan ^{1}(1+2 n)+ \\
8 n^{2} \ln (n) 4 n^{2} \ln \frac{1}{2}+n+n^{2} \div \frac{\ln (1+2 n(1+n))}{n^{2}}
\end{gathered}
$$

where $\mathrm{n}=\sqrt{30 /}$. This expression can in turn be numerically integrated over the blackbody wavelength distribution at a given radiation source temperature to give the total hemispheric absorptance,

$$
=\frac{\frac{{ }_{H}(,)}{\left(e^{C / T} 1\right)^{5}} d}{\frac{1}{\left(e^{C / T} 1\right)^{5}} d},
$$


where $T$ is the source temperature in Kelvin, $\lambda$ is the wavelength in $\mathrm{cm}$, and $C=1.43879$ $\mathrm{cm} \mathrm{K}$. It should be noted that for radiation incident normal to the surface, the total absorptance for all wavelengths simplifies to approximately

$$
=0.576 \sqrt{T} \quad 0.124 \quad T .
$$

Here $T$ is the blackbody source temperature in Kelvin, and $\rho$ is the absorbing surface resistivity in $\Omega$-cm. The total hemispheric absorptance is

$$
B \quad,
$$

where the constant $B$ is approximately 1.3 for very reflective surfaces ${ }^{2}$.

In the case of metals, the surface interaction with reflected radiation occurs in a skin depth much thinner than the wavelengths of the infrared spectrum. For solid surfaces this layer is likely to be "rough" unless it is extremely highly polished. This roughness increases the effective surface resistivity, which is thus higher than the metallic bulk resistivity. It may be that these two resistivity values are only truly equal in the case of liquid metals, when the surface is smooth on a length scale comparable to the skin depth ${ }^{3}$. Because of this, it would not be surprising if equation (6) under-predicts the absorptance of a surface when $\rho$ is assumed to be the bulk metal resistivity.

\section{EXPERIMENTAL SETUP}

\section{Sample Geometry}

Our goal was to suspend a sample length of the MIRI tube inside a blackbody cavity, control the tube temperature at 18 Kelvin, and measure the radiation power it absorbed as a function of the cavity temperature. The challenge was to suspend the tube, measure its temperature, and control its temperature with a heater, while ensuring that it only absorbed radiation from the cavity at its low-absorptance gold-plated surface. Any thermometer or heater elements mounted on this surface would have appeared much blacker than the gold, as would the tube's exposed open ends or any exposed leads or suspension wires. Addressing these issues required an innovative geometry

The blackbody cavity was a thick-walled rectangular aluminum box with blackpainted aluminum honeycomb epoxied to its inside, shown in Figure 1. It was supported inside the $19-\mathrm{cm}$-diameter by $43-\mathrm{cm}$-tall cold volume of a cryostat cooled by a mechanical cryocooler. The box was thermally strapped to the cryostat's cold plate, which ran as cold as 2.5 Kelvin with no intentionally-applied heat load. The box had a thermometer and heater at each end, and its outside was shielded by a multi-layer thermal blanket. By always independently controlling its two ends at the same temperature, we ensured that the box was essentially isothermal.

Previous measurements on similar painted honeycomb indicated that it is extremely black, with total hemispheric emissivity greater than 0.95 over the relevant temperature range ${ }^{4}$. In addition, there is a geometric effect which enhanced the cavity's effective emissivity. The random nature of any reflections off the honeycomb made our configuration a good approximation to the textbook case of concentric cylinders with 
diffuse reflections at the outer cylinder. In such a case the heat absorbed by the tube is the blackbody value indicated by equation (1) multiplied by $(1+\delta)$, where $\delta$ is equal to the product of three small numbers. These numbers are: the ratio of the tube's surface area to the cavity's surface area, the tube's emissivity, and one minus the honeycomb's emissivity. In our configuration $\delta$ was less than $5 \times 10^{-6}$, so we were justified in treating the cavity as a perfect blackbody.

The overall assembly of the sample inside the box is shown in a cross-section view in Figure 2. Cylindrical copper "guards" passed into the box through holes at its top and bottom ends without touching the box. The guards were independently supported and strapped to the cold plate, and their inner ends were flush with the inner surface of the box's honeycomb. Each guard was hollow, with a small hole in its inner end and a larger hole in its outer end. The outer end of each guard was a flange slightly larger than the holes in the box, which served as a radiation baffle for the holes. Disk-shaped caps, bolted to the flanges, served as support and strap attachment locations for the guards. Slots between each flange and cap allowed wires to pass out from the inside of the guard. A small annular Vespel spacer was epoxied inside the hole in each guard's inner end. The inner diameter of these spacers was slightly larger than the sample tube's outer diameter. The inner end of each guard and spacer was covered with a single layer of aluminized Kapton tape. A close-up cross section view of the bottom guard is shown in Figure 3.

The sample tube ran through the box between the centers of the two guards, and it was epoxied to the inner diameter of each spacer. Thus the spacers served as both structural supports and thermal links between the sample and the guards. A heater assembly, consisting of a very thin heater wire wound around a thicker copper wire, ran through the epoxy-filled inside of the sample tube. This configuration distributed the heater power evenly along the sample and kept the sample nearly isothermal, even when the box was at its highest temperatures. A tiny thermometer was epoxied to the outside of one end of the sample tube, located inside one of the guards. Other thermometers were epoxied to the guards' inner walls near the Vespel spacers.

The leads of the thermometers and of the sample heater ran out through the guard slots, in which they were thermally attached with epoxy. A heater for controlling the guard temperature was mounted near each guard on its thermal strap. The bottom guard's strap ran directly to the cryostat's cold plate, while the top guard's strap ran to a thick copper bar that extended up from the cold plate. A thick aluminum shield, bolted to the cold plate's outer edge, surrounded the entire experiment and isolated it from external thermal radiation. A second shield, cooled below 100 Kelvin by the cryocooler's warmer stage, surrounded the cold plate and its shield and further reduced the radiative heat reaching the box and sample.

The significance of this geometry was that it exposed only a fixed gold-plated length of the tube sample to radiation from the box. The spacers, with their shiny coatings and very small areas facing into the box, absorbed very little radiated power compared to the sample and blocked any radiation from entering the copper guards. The guards were virtually isothermal, and the sample's thermometer, leads, and open ends were contained inside the guards. Thus, any radiative or conductive heat flow between the sample and guards occurred inside the guards and was independent of the box 
temperature. This constant heat flow was calibrated in situ, allowing us to determine the absorbed radiation from changes in the sample temperature-control heater power.

The thermometers were read out and the control heaters were powered by PID controllers. We read the sample heater's current and voltage using two multimeters, and data were graphed real-time and archived by a custom computer program.

\section{Pressure Considerations}

To ensure that the measured heat absorbed by the sample tube was due almost exclusively to thermal radiation from the box, it was crucial to keep the gas pressure inside the box extremely low. Using equations found in the literature ${ }^{5}$, we estimated the sample tube heat load which would result from low pressure hydrogen or helium gas inside the box. We found that with either helium at $1 \times 10^{-8}$ Torr or hydrogen at $5 \times 10^{-9}$ Torr, the sample at 18 Kelvin, and the box at 30 Kelvin, the heat reaching the sample from gas conduction was about $10 \%$ of that due to radiation. These numbers assume the eventually-measured sample absorptance value under these conditions. At higher box temperatures this percentage dropped steeply, as the absorbed radiation was proportional to $T^{4}$.

We were unable to directly measure the pressure inside the box, but we took steps to keep it as low as possible. A charcoal getter was installed on the cold plate to ensure that helium and hydrogen gas pressures remained very low, and we assumed that at the lower test temperatures other gases would freeze on the surfaces with no significant vapor pressure. A cold cathode gauge on the cryostat's vacuum shell measured the pressure there, giving typical readings of about $3 \times 10^{-8}$ Torr. Our past experience using a residual gas analyzer on similar cryostats led us to believe that its gas constituents were dominated by nitrogen and water vapor desorbing from the room temperature surfaces.

At the lowest box temperatures, at or below 50 Kelvin, we were concerned about possible steady sources of helium or hydrogen gas, which might maintain a non-zero pressure inside the box despite the getter. We found a published reference to the fact that ion gauges exposed to a significant pressure of water vapor can actually produce a sustained flow of hydrogen gas ${ }^{6}$. We avoided this problem by removing all multi-layer insulation from the cryostat's outer shield and only turning on the pressure gauge for short periods of time before and after the data acquisition. A helium leak detector plumbed into the cryostat at the beginning and end of the run gave an un-measurably low background signal.

When the box temperature was raised above about 140 Kelvin, we worried that water or other gases might desorb from the honeycomb and conduct heat to the sample. To minimize this, we replaced the aluminized Kapton multi-layer blanket from the honeycomb box with four loose layers of aluminum foil, and we held the box at 50 Celsius for four days while pumping on the cryostat before cool-down. During the box's cool-down period, which took about two days, we used the guard strap heaters to hold the guards and sample at room temperature. This avoided the possibility of gas from the high temperature box freezing onto the colder sample surface, and perhaps affecting its absorptance.

While adopting these approaches did not guarantee extremely low pressures, it was the limit of our capability within the scope of the program. From JWST's 
perspective, gas conduction would have indicated a falsely-high absorptance, so the reported results could be considered conservative.

\section{MEASUREMENT TECHNIQUE}

We measured the blackbody power per unit length absorbed by the sample for box temperatures between 35 and 200 Kelvin. For box temperatures up to $130 \mathrm{~K}$, the sample was controlled at 18 Kelvin, its expected operating temperature during the JWST mission. With the box at higher temperatures, the cryostat's cold plate reached a temperature that forced us to hold the sample above 18 Kelvin.

For each sample temperature selected during the test, a corresponding guard temperature was chosen a few Kelvin below that of the sample. For each sample and guard temperature we performed a "zero" power measurement, with the box controlled at the sample temperature. In this configuration there was no radiative heat exchange between the box and sample, and sample heater power alone was conducted through the spacers to the guards. A small amount of heat was conducted to the guards through the sample heater and thermometer leads, and there was even some thermal radiation between the sample and guards. However, all of this all occurred inside the guards, unaffected by the box temperature, and it was included in the zero measurement.

For the actual measurements, the guard and sample temperatures were held constant, the box was controlled at a higher temperature, and we waited for the sample heater power to stabilize. Because the sample was now absorbing radiation from the box, its heater power was lower than during the corresponding zero measurement. The difference between these two sample heater power values was the radiative power absorbed by the sample, which we were able to resolve to a fraction of a $\mu$ Watt. The values of absorbed power per unit length are given in Table 1 and are shown graphically in Figure 4.

We found that 35 Kelvin was the coldest box temperature for which we could reasonably resolve the power absorbed by the 18 Kelvin sample. In this configuration we assumed that there was "grey-body" heat exchange and that $\alpha=\varepsilon$ for the sample. Thus, according to Equation (1), we had

$$
\varepsilon(18 K)=\alpha(35 K, 18 K)=\frac{\dot{Q}}{\sigma A\left(T_{\text {Box }}^{4}-T_{\text {Sample }}^{4}\right)}=0.0079
$$

Here $\dot{Q}$ was the power absorbed by the sample. For all other box temperatures we assumed this value for the sample emissivity, even when it was held at temperatures above $18 \mathrm{~K}$. For these cases, according to Equation (1) we had

$$
\alpha=\frac{\frac{\dot{Q}}{\sigma A}+\varepsilon T_{\text {Sample }}^{4}}{T_{B o x}^{4}}
$$

The sample emissivity term was barely significant even for the lowest box temperatures, but it was included for completeness. The resulting absorptance values are listed in Table 1 and shown graphically in Figure 5. The error bars on the data are due to random 
uncertainty in our determination of the absorbed power. They do not include any possible systematic errors, such as a non-zero helium or hydrogen gas pressure. This is also true of the error bars in Figure 4.

After completing the absorpance measurement, we removed the sample tube and attempted to determine the electrical resistivity of the gold on its surface. The tube's outer diameter was approximately $2 \mathrm{~mm}$, and for convenience we cut it to a length of about $15 \mathrm{~cm}$. According to Epner Technology, Inc., the vendor who applied the gold coating, its mean thickness was determined to be $0.83 \mu \mathrm{m}$ using X-ray florescence. Beneath the gold was a "nickel strike" with average thickness of $0.3 \mu \mathrm{m}$. We used silver epoxy to install current leads to the tube's ends and voltage leads slightly inboard of the ends. We similarly wired up a length of the un-plated stainless steel tubing that had been sent to Epner. We installed these two samples in a cryostat and measured their resistances using a resistance bridge which could resolve one $\mu \Omega$. Comparing the measured resistances of these two samples allowed us to determine the resistance of the gold/nickel coating, and to place lower limits on the possible resistivity of the gold itself.

At 293 Kelvin the un-plated tube's resistance was $63.2 \mathrm{~m} \Omega$, and the plated tube's resistance was $0.5 \mathrm{~m} \Omega$ lower. Taking into account the nominal nickel thickness and accepted room temperature value for nickel's bulk resistivity, the indicated gold resistivity was $1.4 \times 10^{-7} \Omega \cdot \mathrm{m}$, or 6.4 times the accepted value of $2.2 \times 10^{-8} \Omega \cdot \mathrm{m}$ for bulk gold at that temperature ${ }^{7}$. The lowest possible room temperature resistivity for the gold would correspond to the nickel being much thinner than the value claimed by the vendor. Ignoring the nickel altogether and assuming the reported gold thickness was correct, the lowest possible room temperature resistivity for the gold would be $9.7 \times 10^{-8} \Omega \cdot \mathrm{m}$, or 4.4 times the accepted bulk value. We performed the same measurement with the samples at 7 Kelvin, where their resistances were approximately $45 \mathrm{~m} \Omega$. At this temperature, again ignoring the nickel, we found that the lowest possible gold resistivity was $4.8 \times 10^{-8} \Omega \cdot \mathrm{m}$, or more than twice the accepted room temperature bulk value. This value becomes even higher if we take into account a non-zero thickness of nickel under the gold, although we do not know the nickel's 7 Kelvin resistivity. It should be noted that for a gold coating of nearly a $\mu \mathrm{m}$, we would not expect this discrepancy to be a finite-size effect due to the thickness. Thus, it seems that the coating technique results in a higher average resistivity than bulk gold.

\section{DATA ANALYSIS}

We used Mathematica ${ }^{\mathrm{TM}}$ to calculate the total absorptance as a function of source temperature with equations (5) and (6). We varied the surface resistivity, finding that a value of $4.35 \times 10^{-8} \Omega \cdot \mathrm{m}$ produced a good match to our data above about 100 Kelvin. The absorptance theory curve shown in Figure 5 assumes this value, as does the power curve in Figure 4, calculated using equation (10). Below 100 Kelvin the data appear to be systematically higher than the curve, except for the 35 Kelvin value with large error bars. This slight divergence from the theoretical temperature dependence is not understood, but it is qualitatively consistent with low-pressure gas conduction. This will hopefully not be a concern during the JWST mission in the vacuum of space.

For thin metallic coatings it is possible for the effective absorptance to increase at low temperatures due to skin depth effects. The skin depth of a non-magnetic metal surface is well approximated by 


$$
=\sqrt{\frac{2}{0}},
$$

where $\omega$ is the angular frequency, and $\mu_{0}$ is the magnetic permeability of a vacuum. With our best-fit gold resistivity, and the peak blackbody spectrum frequency at 35 Kelvin, the predicted skin depth is only $0.06 \mu \mathrm{m}$. Thus, even at our lowest test temperature, it's unlikely that a significant fraction of the radiation penetrates through the gold and reaches the nickel or stainless steel beneath.

The "best fit" resistivity is strikingly close to the lowest possible bulk resistivity indicated by our resistance measurement. The uncertainty and local variability in the gold thickness is not known. However, if the gold on our sample were moderately thinner than the average value given by Epner, the two resistivity values would be consistent with each other. This suggests that it is mostly the high overall gold resistivity, rather than a skin-depth surface resistivity effect, that leads to the observed absorptance.

\section{CONCLUSION}

We reported the measured absorptance results to the JWST thermal analysis team, and they confirmed that their model now predicts an acceptably low heat absorption by the MIRI cryocooler line during the mission. While this result is not surprising, it is very reassuring to know it from a direct measurement. The gold plating method has been shown to meet the project's needs, but it did not achieve the low absorptance that one would expect from a very smooth bulk gold surface. There is evidence that the gold's average room temperature resistivity is up to six times the accepted value for bulk gold. Unfortunately, investigations into the cause of the gold's higher resistivity and absorptance were beyond the scope of this work.

\section{ACKNOWLEDGEMENTS}

This work was supported by NASA's James Webb Space Telescope program.

\section{REFERENCES}

${ }^{1}$ Eckert, E. R. G., and Drake, R. M., Analysis of Heat and Mass Transfer, McGraw-Hill, New York, 1972, p. 608.

${ }^{2}$ Sparrow, E. M., and Cess, R. D., Radiation Heat Transfer, Hemisphere Publishing Corp., Washington, 1978, p. 71.

${ }^{3}$ Tsujimoto, S., Kanda, M., and Kunitomo, T., "Thermal Radiative Properties of Some Cryogenic Materials," Cryogenics, 1982, pp. 591-597.

${ }^{4}$ Tuttle, J., et al., "The Total Hemispheric Emissivity of Painted Aluminum Honeycomb at Cryogenic Temperatures," in Advances in Cryogenic Engineering 59A, edited by J. G. Weisend et al., AIP Conference Proceedings 1573, American Institute of Physics, Melville, NY, 2014, pp. 590-596. 
${ }^{5}$ Dushman, S., Scientific Foundations of Vacuum Technique, Wiley \& Sons, New York, 1962, pp. 43-53.

${ }^{6}$ Mukugi, K., Tsuchidate, H., and Oishi, N., "Characteristics of Cold Cathode Gauges for Outgassing Measurements in UHV Range,” Vacuum, Vol. 44, 1993, pp. 591-593.

${ }^{7}$ Matula, R. A., "Electrical Resistivity of Copper, Gold, Palladium, and Silver," J. Phys. Chem. Ref. Data, Vol. 8, No. 4, 1979, pp. 1147-1298. 


\section{FIGURE CAPTIONS}

Figure 1. The honeycomb box with its bottom removed. The top, identical to the bottom, is not shown.

Figure 2. A cross-sectional schematic view of the test set-up. For clarity, details of the cryostat and blanketing are not shown.

Figure 3. A cross-sectional schematic view of the bottom guard and the bottom end of the sample tube assembly. The heater, coiled around a copper wire, can be seen inside the sample tube.

Figure 4. The power absorbed by the sample per unit length, as a function of the blackbody source temperature. The solid circles are values measured with the sample tube at 18 Kelvin. For the open circles the sample tube was at temperatures between $18.4 \mathrm{~K}$ and $38.2 \mathrm{~K}$. The line is a theoretical curve, calculated from equations (5), (6), and (10) assuming the electrical resistivity of the gold coating was $4.35 \times 10^{-8} \Omega-\mathrm{m}$.

Figure 5. The measured absorptance of the sample. The solid circles are values measured with the sample tube at 18 Kelvin. For the open circles the sample tube was at temperatures between $18.4 \mathrm{~K}$ and $38.2 \mathrm{~K}$. The line is a theoretical curve, calculated from equations (5) and (6) assuming the electrical resistivity of the gold coating was $4.35 \times 10^{-8} \Omega-\mathrm{m}$.

Table 1 . The power absorbed by the sample tube per unit length, and the corresponding calculated absorptance. 


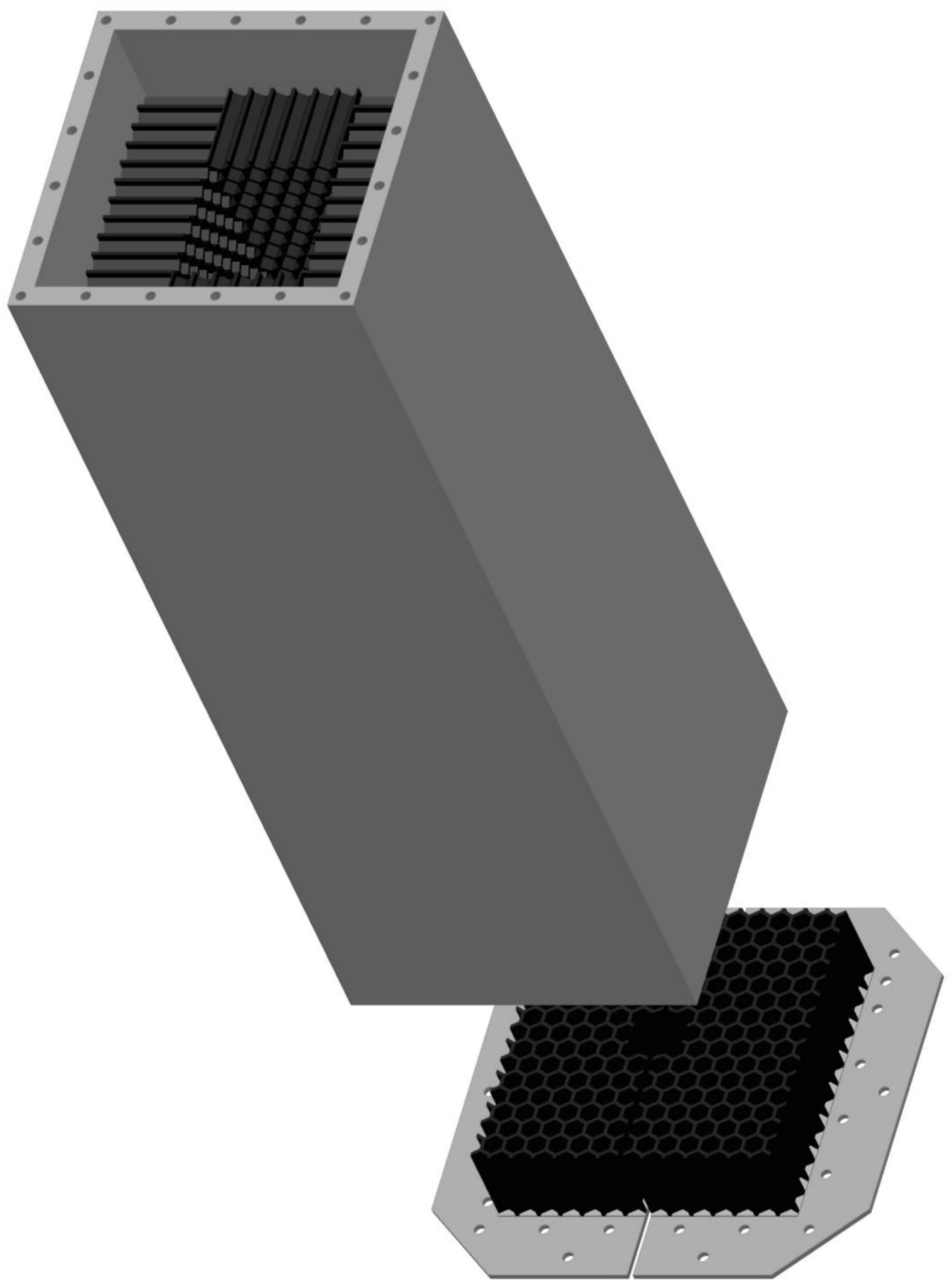

Figure 1. The honeycomb box with its bottom removed. The top, identical to the bottom, is not shown 


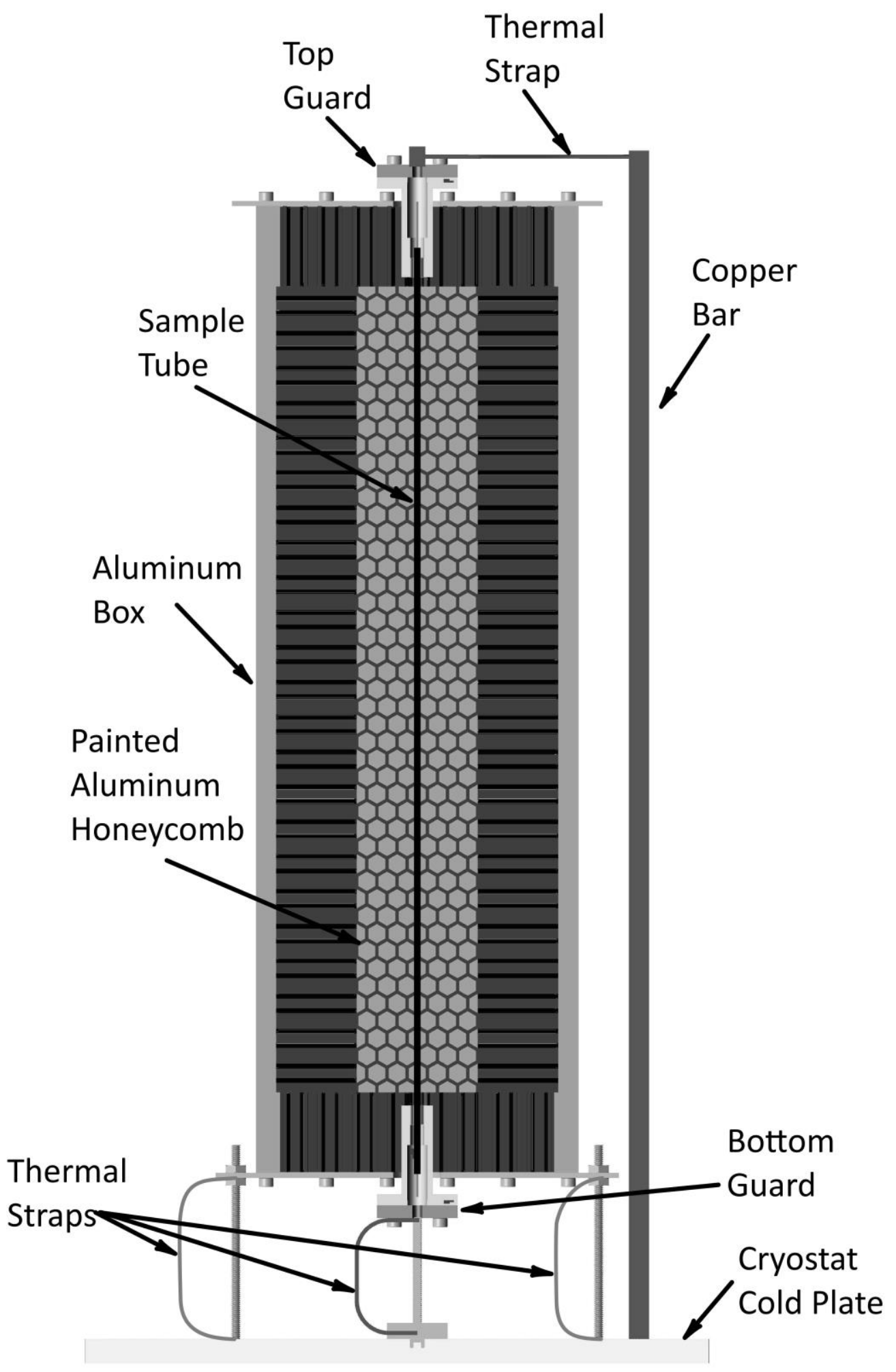

Figure 2. A cross-sectional schematic view of the test set-up. For clarity, details of the cryostat and blanketing are not shown. 


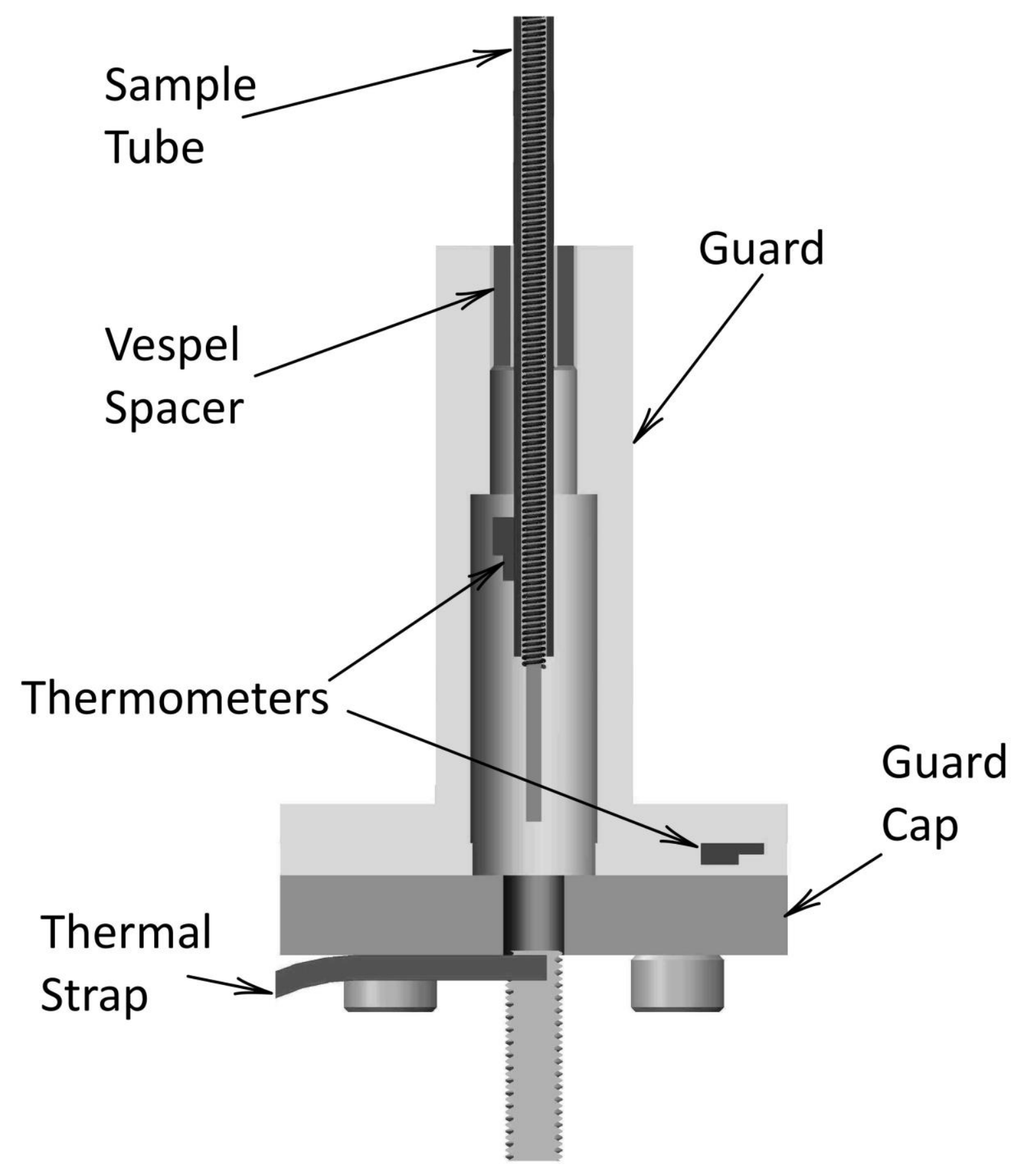

Figure 3. A cross-sectional schematic view of the bottom guard and the bottom end of the sample tube assembly. The heater, coiled around a copper wire, can be seen inside the sample tube. 


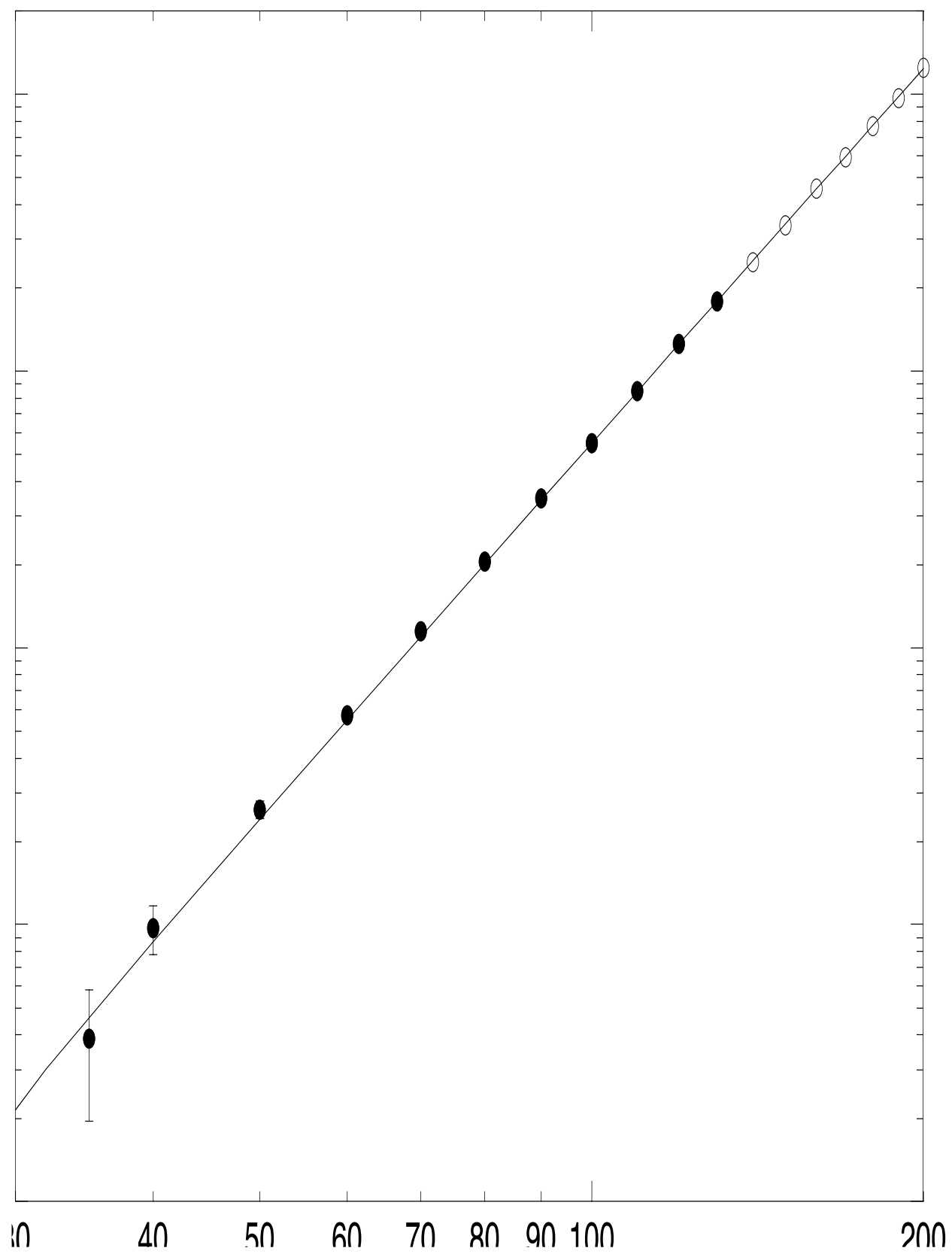

Figure 4. The power absorbed by the sample per unit length, as a function of the blackbody source temperature. The solid circles are values measured with the sample tube at 18 Kelvin. For the open circles the sample tube was at temperatures between $18.4 \mathrm{~K}$ and 38.2 $\mathrm{K}$. The line is a theoretical curve, calculated from equations (5), (6), and (10) assuming the electrical resistivity of the gold coating was $4.35 \times 10^{-8} \Omega-\mathrm{m}$. 


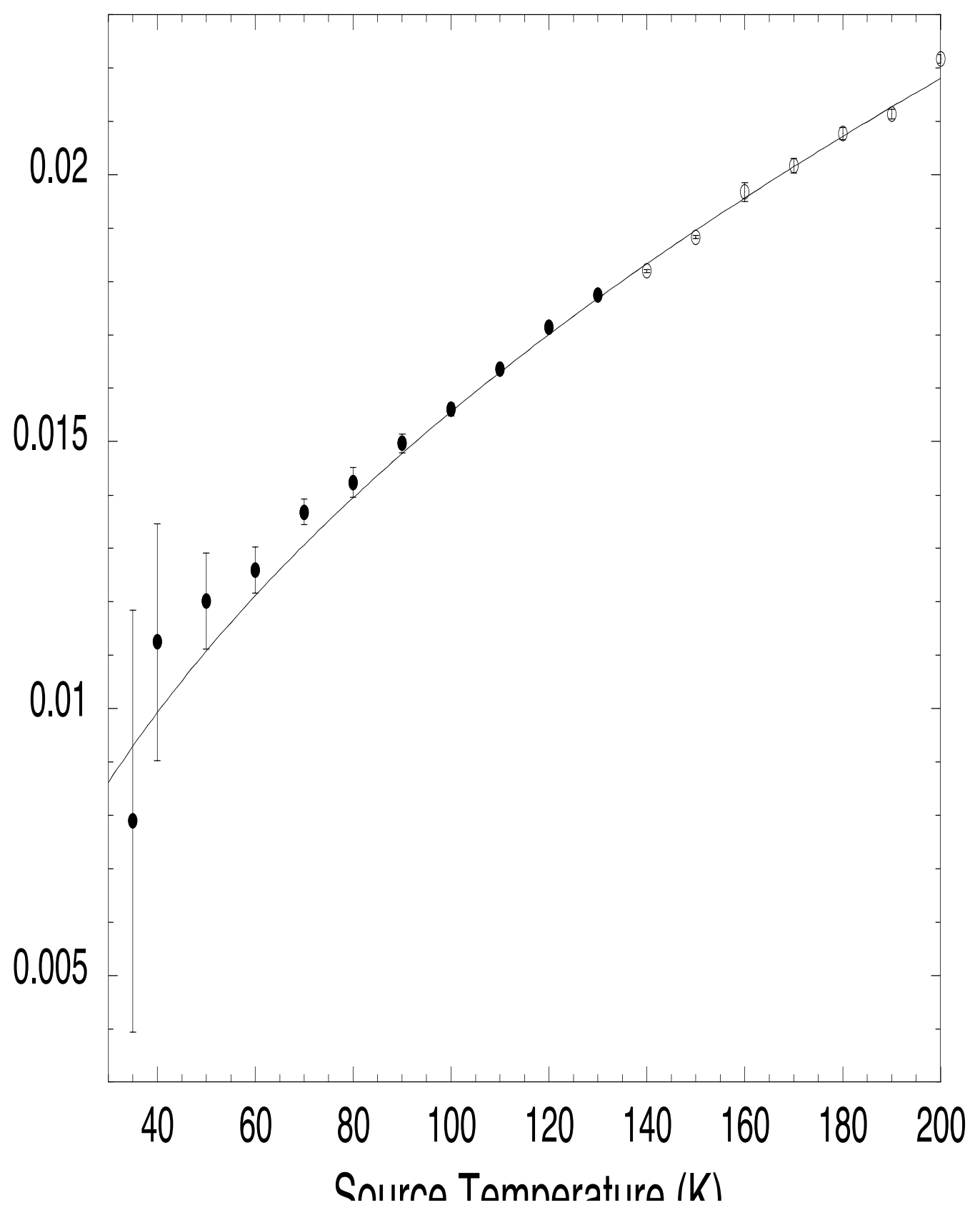

Figure 5. The measured absorptance of the sample. The solid circles are values measured with the sample tube at 18 Kelvin. For the open circles the sample tube was at temperatures between $18.4 \mathrm{~K}$ and $38.2 \mathrm{~K}$. The line is a theoretical curve, calculated from equations (5) and (6) assuming the electrical resistivity of the gold coating was $4.35 \times 10^{-8} \Omega-\mathrm{m}$. 


\begin{tabular}{|c|c|c|c|}
\hline $\begin{array}{c}\text { Sample T } \\
(\mathrm{K})\end{array}$ & $\begin{array}{c}\text { Blackbody T } \\
(\mathrm{K})\end{array}$ & $\begin{array}{c}\text { Absorbed } \\
\text { Power/Length } \\
(\mathrm{W} / \mathrm{m})\end{array}$ & Absorptance \\
\hline 18.0 & 35 & $3.89 \mathrm{E}-06$ & 0.00790 \\
\hline 18.0 & 40 & $9.72 \mathrm{E}-06$ & 0.01108 \\
\hline 18.0 & 50 & $2.61 \mathrm{E}-05$ & 0.01195 \\
\hline 18.0 & 60 & $5.72 \mathrm{E}-05$ & 0.01256 \\
\hline 18.0 & 70 & $1.15 \mathrm{E}-04$ & 0.01367 \\
\hline 18.0 & 80 & $2.05 \mathrm{E}-04$ & 0.01422 \\
\hline 18.0 & 90 & $3.46 \mathrm{E}-04$ & 0.01496 \\
\hline 18.0 & 100 & $5.50 \mathrm{E}-04$ & 0.01560 \\
\hline 18.0 & 110 & $8.45 \mathrm{E}-04$ & 0.01635 \\
\hline 18.0 & 120 & $1.25 \mathrm{E}-03$ & 0.01714 \\
\hline 18.0 & 130 & $1.79 \mathrm{E}-03$ & 0.01775 \\
\hline 18.4 & 140 & $2.47 \mathrm{E}-03$ & 0.01820 \\
\hline 21.0 & 150 & $3.36 \mathrm{E}-03$ & 0.01883 \\
\hline 23.5 & 160 & $4.55 \mathrm{E}-03$ & 0.01968 \\
\hline 25.8 & 170 & $5.94 \mathrm{E}-03$ & 0.02016 \\
\hline 29.2 & 180 & $7.69 \mathrm{E}-03$ & 0.02077 \\
\hline 33.6 & 190 & $9.72 \mathrm{E}-03$ & 0.02113 \\
\hline 38.2 & 200 & $1.25 \mathrm{E}-02$ & 0.02217 \\
\hline
\end{tabular}

Table 1. The power absorbed by the sample tube per unit length, and the corresponding calculated absorptance. 